\title{
Fibronectin 1: A Potential Biomarker for Ovarian Cancer
}

\author{
Huijing Bao $\mathbb{D},{ }^{1,2}$ Qianyu Huo, ${ }^{2}$ Qin Yuan, ${ }^{2}$ and Chen Xu $\mathbb{D}^{3}$ \\ ${ }^{1}$ The Integrative Medical Diagnosis Laboratory, The Tianjin Nankai Hospital, Tianjin, China 300100 \\ ${ }^{2}$ School of Laboratory Science, Tianjin Medical University, Tianjin, China 300203 \\ ${ }^{3}$ The Department of Laboratory Science, Tianjin 4th Central Hospital, Tianjin, China 300140 \\ Correspondence should be addressed to Chen Xu; skyalong1028@126.com
}

Received 1 February 2021; Revised 26 April 2021; Accepted 11 May 2021; Published 22 May 2021

Academic Editor: Ferdinando Mannello

Copyright (c) 2021 Huijing Bao et al. This is an open access article distributed under the Creative Commons Attribution License, which permits unrestricted use, distribution, and reproduction in any medium, provided the original work is properly cited.

\begin{abstract}
Object. Ovarian cancer is one of the most common cancers among females with high mortality rate, due to most patients diagnosed at the advanced stage of the disease. Seeking new biomarkers for ovarian cancer detection and progress indication is really important for the patients. Methods. OVCAR3 and A2780 are the two common cell lines that are used for ovarian cancer studies. The different invasion and migration abilities were observed by scratch tests and transwell experiments in our preliminary study. Gene chip was used to screen the expression gene in these two different cell lines, and then, the differentially expressed genes (at least 2-fold difference, $P$ value $<0.05$ ) were analyzed using KEGG. Result. Fibronectin 1 (FN1) was found to be the most strongly correlated with the invasion and migration abilities of the OVCAR3 cells. Real-time PCR and FN1 knockout cell line was conducted and confirmed this finding. Based on the Oncomine database analysis, comparing with normal people, ovarian cancer patients exhibited high levels of FN1 expression. Additionally, higher FN1 expression was found in patients with higher FIGO stages of cancer. Conclusion. FN1 could be a new biomarker for ovarian cancer detection and progress indicator.
\end{abstract}

\section{Introduction}

Ovarian cancer is the fifth leading cause of cancer-related deaths among women worldwide; unfortunately, early detection tests are relatively lacking. Furthermore, most women with ovarian cancer are diagnosed in the late stages of the disease, which portends a poor prognosis [1,2]. Current surveillance strategies, which are achieved by transvaginal ultrasound or serum tumor marker cancer antigen 125 (CA125) tests, are ineffective in detecting ovarian cancer at an early stage $[3,4]$. Therefore, the early detection and diagnosis of ovarian cancer have a very important role in the survival and prognoses of patients with epithelial ovarian cancer.

Fibronectin (FN) is a high-molecular-weight glycoprotein of the extracellular matrix that mediates a wide variety of cellular interactions with the extracellular matrix (ECM) and plays important roles in cell adhesion, migration, growth, and differentiation $[5,6]$. FN1 is a member of the FN family, which has different functions during a variety of biological processes including cell adhesion, cell migration, and cytoskeletal organization in many different diseases [7]. In a variety of tumors, such as nasopharyngeal carcinoma, osteosarcoma, esophageal cancer, and ovarian cancer, FN1 is an important tumor-related gene [8-11].

Currently, the cell lines A2780 and OVCAR3 are commonly used in many different studies of ovarian cancer [12, 13], but no examinations of the differences between these two cell lines have been reported. In our study, greater migration and invasion abilities were found in the OVCAR3 cells compared with the A2780 cells. Therefore, we want to see if good protein candidates could be used as diagnostic markers for ovarian cancer based on these phenomena.

\section{Materials and Methods}

2.1. Cell Lines and Cell Culture. A2780, Caov3, and SKOV3 cells (CoBioer, China) were incubated in RPMI 1640 supplemented with $10 \%$ fetal bovine serum (Gibco, USA). OVCAR3 cells (CoBioer, China) were cultured in medium containing RPMI 1640, 10\% fetal bovine serum, and 1\% insulin (Sigma, China). 
2.2. Cell Scratch Test. Cells were seeded into six-well plates, and when the density reached $100 \%$, a scratch was made through the cells. Photographs were taken at $48 \mathrm{~h}, 72 \mathrm{~h}$, or $96 \mathrm{~h}$ after the scratch using an inverted tissue culture microscope (Nikon ECLIPSE Ti-U) at $\times 4$ magnification.

2.3. Transwell Migration Assay. The two ovarian cancer cell lines, i.e., A2780 and OVCAR3 $\left(5 \times 10^{4}\right.$ cells/well), were seeded on transwell inserts (polycarbonate filters with $8 \mu \mathrm{m}$ pores, Costor) filled with medium. Medium $(600 \mu \mathrm{l})$ was added to the bottom chamber. After $48 \mathrm{~h}$ of culture at $37^{\circ} \mathrm{C}$ under humidified 5\% $\mathrm{CO}_{2}$ in air, the cells that remained inside the upper chamber were gently and completely removed with a cotton swab. The cells that migrated to the lower surface of the filter membrane were fixed with anhydrous methanol and stained with $0.1 \%$ crystal violet. The filters were air dried, and photographs were collected with an inverted phasecontrast microscope (Nikon ECLIPSE Ti-U). After collecting the photographs, the membrane was washed with $500 \mu \mathrm{l}$ $50 \%$ aqueous acetic acid solution for approximately $5 \mathrm{~min}$, $100 \mu \mathrm{l}$ of an eluent was added to a clean 96-well plate, and the OD values were measured at $595 \mathrm{~nm}$ using an ELISA reader (Synergy 2, China).

2.4. Microarray Analyses of the Nucleic Acids. Microarray analyses of the nucleic acids were performed using Affymetrix ${ }^{\circledR}$ Human Genome U219 Array Stripes (Affymetrix, USA) according to the manufacturer's instructions. The procedure included genomic DNA extraction fromA2780 or OVCAR3, digestion and ligation, PCR amplification, PCR product purification, quantification and fragmentation, labeling, array hybridization, washing, and scanning. Gene expression analyses were performed using the Affymetrix ${ }^{\circledR}$ Transcriptome Analysis Console (TCA) software, which evaluates the expressions of different genes. The Kyoto Encyclopedia of Genes and Genomes (KEGG) was also used for subsequent related pathway analysis.

2.5. Fluorescence Quantitative Real-Time PCR. RNA was isolated from each sample using TRIzol reagent (Ambion, USA) according to the manufacturer's instructions. First-strand cDNA was synthesized from the total RNA using oligo (dT) primers from the FastQuant RT Kit. Real-time PCR was performed on a stratagene MX3005P. Glyceraldehyde-3phosphate dehydrogenase (GAPDH) was used as an internal control. The SYBR Green PCR Master Mix Reagent Kit was utilized for the PCRs, and the relative gene expressions were determined based on the threshold cycles $(\mathrm{Ct})$ of the target gene and the internal reference gene. The average $\mathrm{Ct}$ value of the GAPDH gene was subtracted from the average $\mathrm{Ct}$ value of each target gene. The fold change $\left(2^{-\Delta \Delta \mathrm{Ct}}\right)$ in expression was calculated for the gene of interest relative to the internal control gene (GAPDH) for each of the analyzed cancer cell lines. The primer sequences used in PCRs were as follows: FN1 sense: $5^{\prime}$-GTTCGGGAGGAGGTTGTTA CC-3' and antisense: $5^{\prime}$-GAGTCATCTGTAGGCTGGTTT AGG-3' and GAPDH: sense: $5^{\prime}$-CCTCCAAGGAGTAAGA CCCC- $3^{\prime}$ and antisense: $5^{\prime}$-AGGGGTCTACATGGCA ACTG-3'. Each sample was run in triplicate.
2.6. FN1 KO Cell Construction. 293T cells were seeded on the plate, and then, the cells were cultured when the fusion degree was up to $80 \%$. Lenti-CRISPR-V2-sgRNA-FN1 or lentiCRISPR-V2, psPAX2, and pMD2.G were mixed into $1 \mathrm{ml}$ Opti-MEM medium under a ratio of $4: 3: 1$ and then incubated at room temperature for $5 \mathrm{~min} .30 \mu \mathrm{l}$ PEI was added into the mixture, and then, the mixture was incubated at room temperature for $30 \mathrm{~min}$. After incubation, the mixture was added to $293 \mathrm{~T}$ cells and incubated at $37^{\circ} \mathrm{C}$ for $6 \mathrm{~h}$ with $5 \%$ $\mathrm{CO}_{2}$. After $48 \mathrm{~h}$, the cell culture medium was changed to low serum medium (4\% FBS), and the virus packaging supernatant was collected and stored at $-80^{\circ} \mathrm{C}$.

OVCAR3 cells were incubated for $24 \mathrm{~h}$ before virus transfection, and then, virus packaging supernatant with the final concentration of $10 \mu \mathrm{g} / \mathrm{ml}$ polybrene mixed with complete medium under $1: 1$ ratio was performed into the OCVAR3 cells. After 36 hours of continuous culture, $0.2 \mu \mathrm{g} / \mathrm{ml}$ puromycin was added into the medium for FN1 KO cell screening.

2.7. Western Blotting. Cells were washed in PBS and lysed in SDS loading buffer (KeyGEN BioTECH, KGP101) and subjected to the SDS-PAGE gel. The proteins extracted from the cells were transferred onto the PVDF membrane (Millipore, ISEQ00010). Membranes were blocked with 5\% $\mathrm{w} / \mathrm{v}$ milk (0.1\%TBST) and incubated with mouse anti-FN1 antibody (affinity, BF0273, 1:1000) and mouse anti-betatubulin antibody (affinity, T0023, $1: 2000$ ) overnight in $4^{\circ} \mathrm{C}$. Secondary antibody (affinity, S0002, $1: 1000$ ) was incubated with membrane for $1 \mathrm{~h}$ at room temperature.

2.8. ELISA. The experiment was conducted by the manuscript protocol (LiuHe, LH-E10073HU).

2.9. Statistical Analysis. SPSS software was used for statistical analysis. The results are expressed as the mean \pm SD or the mean \pm SEM. Two-tailed unpaired Student's $t$-tests were used to determine the statistical significance, and $P<0.05$ was considered statistically significant.

\section{Results}

3.1. Detecting the Difference of Migration and Invasion Abilities. To determine the differences of the migration and invasion abilities of these two ovarian cancer cell lines, scratch and transwell experiments were performed. The cells were seeded in 6-well plates, and the migration abilities were measured at $0 \mathrm{~h}, 48 \mathrm{~h}, 72 \mathrm{~h}$, and $96 \mathrm{~h}$. Compared with those in the A2780 group, the diameters of the scratches became narrow in the OVCAR3 group (Figure 1(a)). This difference indicated that the OVCAR3 cells exhibited stronger migration ability than A2780 cells. The invasion abilities were determined with the transwell experiments, and the numbers of invasive cells were counted at $48 \mathrm{~h}$ after the cells were seeded. The results revealed that a much greater number of invasive cells were observed in the OVCAR3 group (Figure 1(b)). This conclusion was also confirmed by the $595 \mathrm{~nm}$ OD reading results after the invasive cells were dissolved in 50\% aqueous acetic acid solution (Figure 1(c)). Based on these results, the migration and invasion abilities 


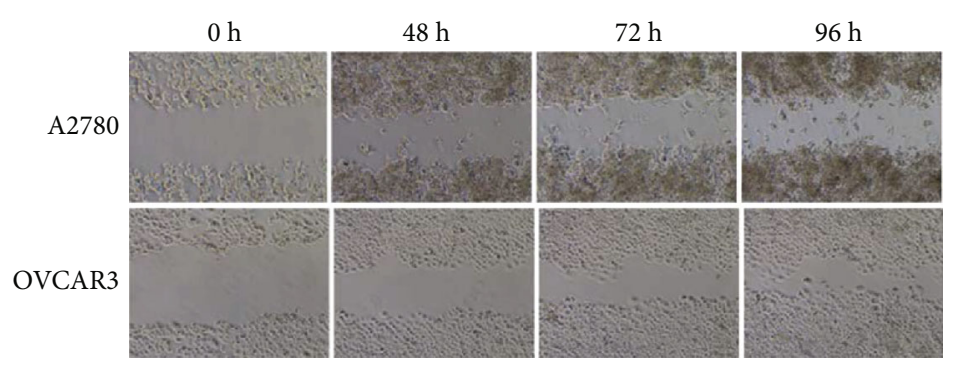

(a)

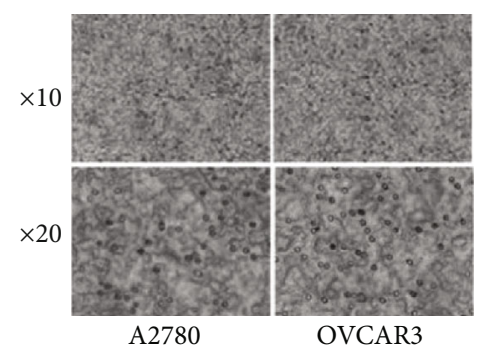

(b)

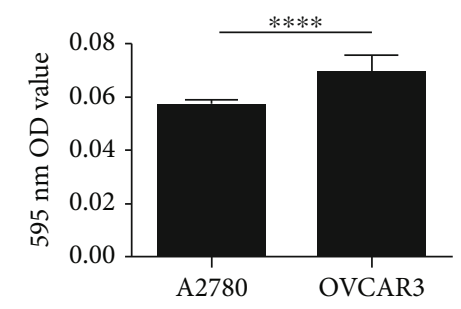

(c)

FIGURE 1: The migration capabilities of the A2780 and OVCAR3 cell lines were detected with scratch tests and transwell migration assays. (a) A wound healing assay was performed to assess the migration capacities of A2780 and OVCAR3 cells. (b) Transwell migration assays of the A2780 and OVCAR3 cells showing their migration abilities. (c) Quantitative results of the transwell invasion assays. Significant differences were identified using unpaired $t$-test analyses and are indicated by asterisks; ${ }^{* * *} P<0.0001$.

of the OVCAR3 cells were much greater than those of the A2780 cells.

\subsection{Determining the Different mRNA Expressions. To deter-} mine the cause of the differences between these two cell lines, gene chip analysis was conducted with the OVCAR3 and A2780 cells. Many differentially expressed genes were found in these two ovarian cancer cell lines (Figures 2(a) and 2(b)) based on analyses of the volcano map results and the hierarchical clustering results. Additionally, the gene expressions of these two cell lines were analyzed in terms of the following four different gene ontology (GO) aspects: molecular function, biological processes, cellular components, and protein (Figure 2(c), continued). The results indicated that the two types of cells, i.e., A2780 and OVCAR3, exhibited significant differences both in gene expression and cellular biology.

3.3. Potential Protein Candidate Analysis. To identify different proteins, cell pathway analysis was performed using the Kyoto Encyclopedia of Genes and Genomes (KEGG). The differentially expressed genes between the two cell lines are presented in Table 1 . The pathway with the greatest number of differences was the focal adhesion pathway. Moreover, some candidates genes $(P<0.0001$ and fold change $\geq 100)$ among the focal adhesion-related genes (i.e., CCND2, ITGB8, and FN1) were selected for further analysis (Figure 3(a)). Statistical analyses of the expressions of these three genes revealed that the difference in FN1 expression was the most statistically significant (Figure 3(b)). To confirm the array results, real-time PCR was used to verify the difference in FN1 expression between the OVCAR3 and A2780 cells. The results revealed that the OVCAR3 cells did express FN1 at a higher level than the A2780 cells (Figure 3(c)), which proved that FN1 plays an important role in cell migration and that a high level of FN1 expression indicates high tumor cell mobility.

To further confirm the results, FN1 KO cell line was constructed by CRISPR. Comparing the four different ovarian cancer cells, high FN1 expression was found in OVCAR3 at mRNA level (Figure 4(a)), protein level (Figure 4(b)), and the cell medium level (Figure 4(c)). So OVCAR3 FN1 KO cell line (V2-sgRNA-FN1) was conducted and confirmed at mRNA level (Figure 4(d)) and protein level (Figure 4(e)).

The differences in the migration and invasion abilities among the wild-type group, the control group (empty vector V2), and the KO group were detected by transwell experiments. The cells were seeded in 6-well plates, and the migration abilities were measured at $48 \mathrm{~h}$. Compared with both the wild-type group and the control group, a significant less number of invasive cells were observed in the KO group (Figures 4(f) and 4(g)). Based on these results, FN1 should be a very important protein for the migration and invasion abilities of the ovarian cancer cells.

3.4. FN1 Clinical Feasibility Evaluation. Clinical samples were used for a FN1 feasibility evaluation. According to the above results, stronger invasion and migration abilities are associated with greater FN1 expression. This relationship indicates that FN1 expression could be used as a good indicator for disease progress.

To examine this possibility, the FN1 expression levels in patients with clinical ovarian cancer were identified using the Oncomine database with set conditions of $P<0.05$ and a fold change of $>3$ or $<-3$. This analysis revealed that different pathological types of ovarian tissue exhibited different FN1 expression levels; ovarian mucinous adenocarcinomas (Figure 5(a)), ovarian clear cell adenocarcinomas (Figure 5(b)), ovarian endometrioid adenocarcinomas (Figure 5(c)), and ovarian 


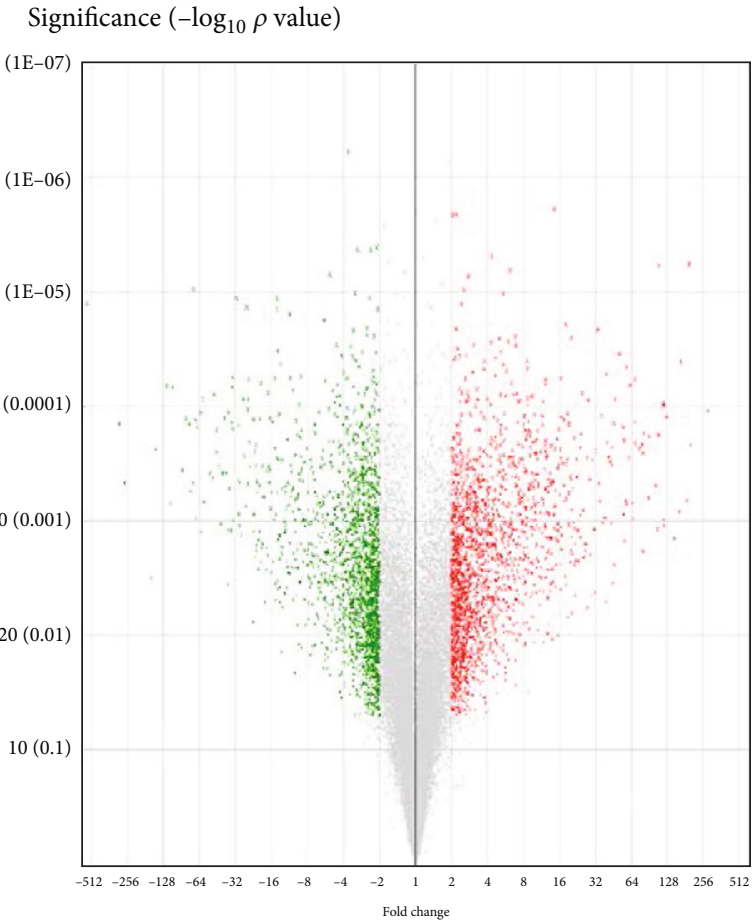

(a)

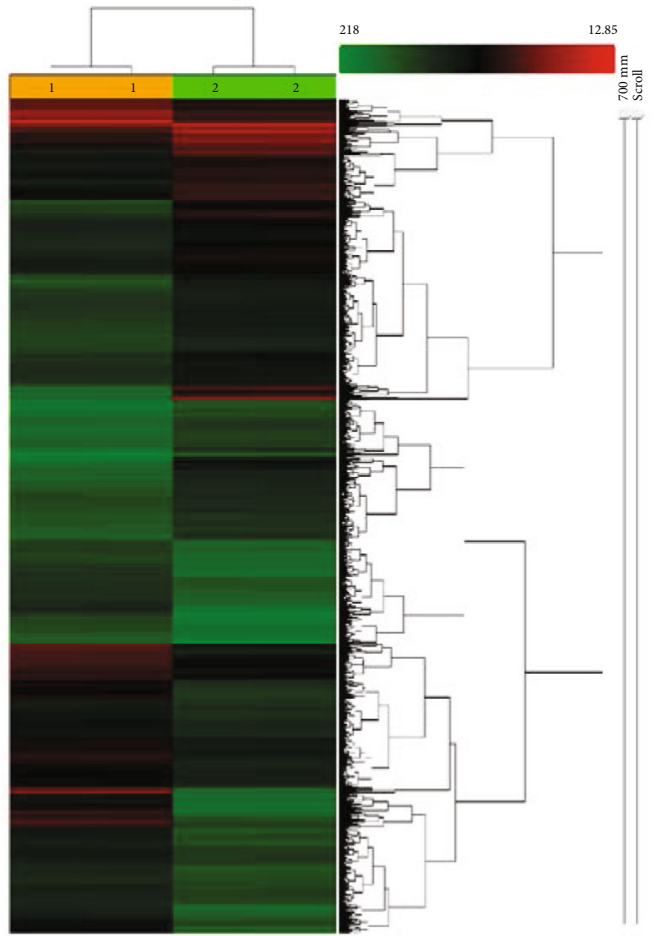

(b)

Figure 2: Continued. 
PANTHER GO-Slim molecular function Total \# genes: 2533 Total \# function hits: 1913

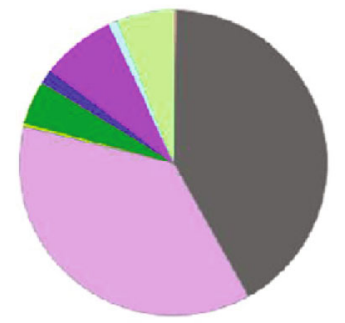

- Antioxidant activity $0.3 \%$

- Binding $41.6 \%$

- Catalytic activity $37.0 \%$

- Channel regulator activity $0.3 \%$

- Receptor activity $7.7 \%$

- Signal transducer activity $1.5 \%$

- Structural molecule activity $7.7 \%$

Translation regulator activity $0.9 \%$

12 Transporter activity $6.0 \%$
PANTHER GO-Slim biological process

Total \# genes: 2533 Total \# process hits: 3825
PANTHER GO-Slim cellular component Total \# genes: 2533 Total \# component hits: 1604

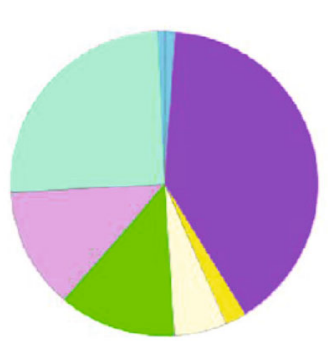

Cell junction $1.1 \%$

- Cell part $40 \%$

- Extracellular matrix 2.1\% Extracellular region 5.6\%

- Macromolecular complex $12.3 \%$

- Membrane $12.9 \%$

Organelle 25.2\%

- Synapse $0.6 \%$

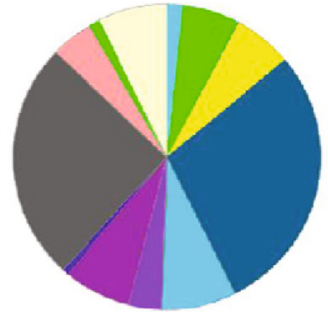

- Biological adhesion $1.7 \%$

- Biological regulation 6.0\% Cell killing 0.1\%

- Cellular component organization or biogenesis $6.2 \%$

- Cellular process $28.8 \%$

Developmental process $7.7 \%$

Growth $0.1 \%$

- Immune system process $3.4 \%$

- Localization $7.3 \%$

- Locomotion $0.6 \%$

- Metabolic process $25.3 \%$

- Multicellular organismal process $4.5 \%$

- Reproduction $1.2 \%$ Response to stimulus $7.2 \%$
PANTHER protein class

Total \# genes: 2533 Total \# protein class hits: 2234

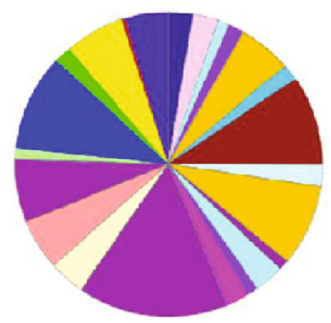

- Calcium-binding protein $2.6 \%$

- Cell adhesion molecule 2.6\%

Cell junction protein $1.2 \%$

- Chaperone $1.6 \%$

- Cytoskeletal protein $5.7 \%$

- Defence/immunity protein $1.8 \%$

n Enzyme modulator 9.5\%

Extracellular matrix protein $2.3 \%$

- Hydrolase $8.6 \%$

- Isomerase $1.1 \%$

Ligase $3.1 \%$

- Lyase $1.2 \%$

- Membrane traffic protein $2.4 \%$

- Nucleic acid binding $15.9 \%$

Oxidoreductase $3.9 \%$

- Receptor 5.3\%

n Signaling molecule $6.6 \%$

Storage protein $0.1 \%$

- Structural protein $1.0 \%$

- Transcription factor $10.3 \%$

- Transfer/carrier protein $1.7 \%$

- Transferase $6.5 \%$

- Transmembrane receptor regulatory/adaptor protein $0.4 \%$

- Transporter $4.4 \%$

(c)

FIGURE 2: The gene expression profiles of the A2780 and OVCAR3 cell lines were analyzed with gene chips. (a) Volcano plot analyses showing the differentially expressed genes between the A2780 and OVCAR3 cells. The $x$-axis represents the $\log _{2}$ (fold change) value, and the $y$-axis represents the $-\log _{10} P$ value. (b) Hierarchical clustering analysis of the differentially expressed genes. (c) Results of the gene ontology analysis. The percent followed by the category name is the percent of a gene hit against the total genes.

serous adenocarcinomas (Figure 5(d)) exhibited higher FN1 expression levels than normal samples. The FN1 expression levels in patients in different FIGO stages were also analyzed. The results revealed that the FN1 expression levels in stage III were significantly greater than those in the low FIGO stages (stages I and II). This result indicated that FN1 expression is positively related to FIGO stage, i.e., greater FN1 expression is associated with a higher FIGO stage.

Taken together, our results indicated that FN1 could be used as a marker for aggressive ovarian cancer detection and can also be applied as an indicator of poor progression for the patients. 
TABle 1: Pathway analysis of the differentially expressed genes (at least 2 -fold change, $P$ value $<0.05$ ) using KEGG.

\begin{tabular}{|c|c|c|}
\hline KEGG ID & Pathway name & $P$ value \\
\hline hsa04510 & Focal adhesion & $4.63 E-08$ \\
\hline hsa04512 & ECM-receptor interaction & $2.77 E-07$ \\
\hline hsa05206 & MicroRNAs in cancer & $1.98 E-05$ \\
\hline hsa05412 & Arrhythmogenic right ventricular cardiomyopathy (ARVC) & $3.21 E-05$ \\
\hline hsa05202 & Transcriptional misregulation in cancer & $7.83 E-05$ \\
\hline hsa05134 & Legionellosis & $9.45 E-05$ \\
\hline hsa04115 & p53 signaling pathway & $1.02 E-04$ \\
\hline hsa04151 & PI3K-Akt signaling pathway & $4.32 E-04$ \\
\hline hsa04360 & Axon guidance & $4.90 E-04$ \\
\hline hsa04350 & TGF-beta signaling pathway & $6.73 E-04$ \\
\hline hsa04670 & Leukocyte transendothelial migration & $8.52 E-04$ \\
\hline hsa05146 & Amoebiasis & 0.001178 \\
\hline hsa05200 & Pathways in cancer & 0.001287 \\
\hline hsa04514 & Cell adhesion molecules (CAMs) & 0.001404 \\
\hline hsa05132 & Salmonella infection & 0.002408 \\
\hline hsa05222 & Small cell lung cancer & 0.002838 \\
\hline hsa04520 & Adherens junction & 0.003269 \\
\hline hsa05205 & Proteoglycans in cancer & 0.004471 \\
\hline hsa05203 & Viral carcinogenesis & 0.005616 \\
\hline hsa04310 & Wnt signaling pathway & 0.009113 \\
\hline hsa04550 & Signaling pathways regulating pluripotency of stem cells & 0.010103 \\
\hline hsa05220 & Chronic myeloid leukemia & 0.012857 \\
\hline hsa04062 & Chemokine signaling pathway & 0.013767 \\
\hline hsa04390 & Hippo signaling pathway & 0.017097 \\
\hline hsa05166 & HTLV-I infection & 0.018198 \\
\hline hsa04068 & FoxO signaling pathway & 0.019317 \\
\hline hsa04666 & Fc gamma R-mediated phagocytosis & 0.027802 \\
\hline hsa05120 & Epithelial cell signaling in Helicobacter pylori infection & 0.029704 \\
\hline hsa04668 & TNF signaling pathway & 0.032627 \\
\hline hsa05218 & Melanoma & 0.038013 \\
\hline hsa04932 & Nonalcoholic fatty liver disease (NAFLD) & 0.039736 \\
\hline hsa05164 & Influenza A & 0.042531 \\
\hline hsa04621 & NOD-like receptor signaling pathway & 0.04312 \\
\hline hsa04530 & Tight junction & 0.051741 \\
\hline hsa05219 & Bladder cancer & 0.054701 \\
\hline hsa04015 & Rap1 signaling pathway & 0.066471 \\
\hline hsa04810 & Regulation of actin cytoskeleton & 0.068376 \\
\hline hsa05142 & Chagas disease (American trypanosomiasis) & 0.072983 \\
\hline hsa04931 & Insulin resistance & 0.085386 \\
\hline hsa04540 & Gap junction & 0.088773 \\
\hline hsa04974 & Protein digestion and absorption & 0.088773 \\
\hline hsa04380 & Osteoclast differentiation & 0.089895 \\
\hline hsa00512 & Mucin type O-glycan biosynthesis & 0.093882 \\
\hline hsa04662 & B cell receptor signaling pathway & 0.094578 \\
\hline hsa05160 & Hepatitis C & 0.096068 \\
\hline
\end{tabular}




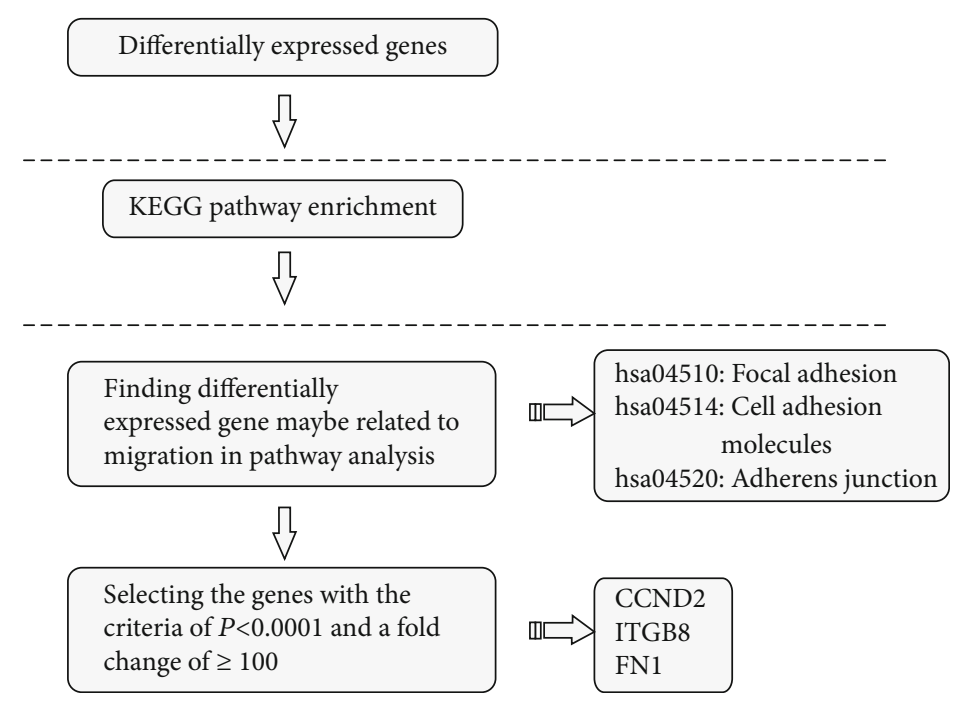

(a)

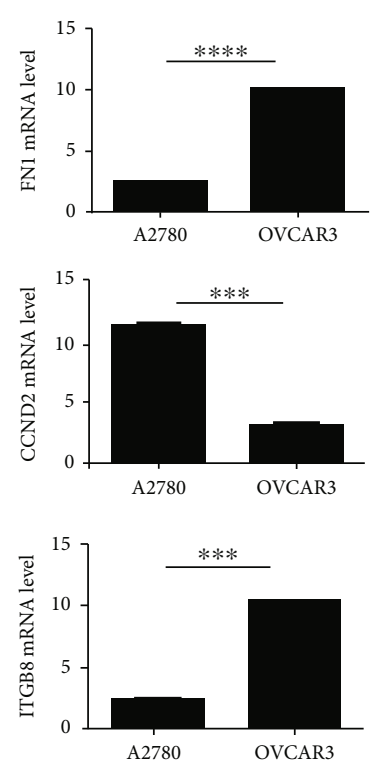

(b)

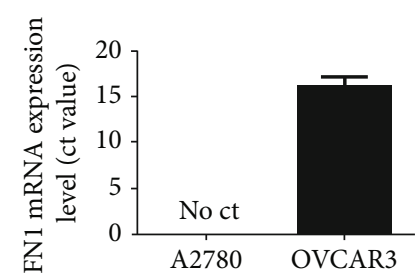

(c)

FIGURE 3: (a) Bioinformatics pipeline used to screen the candidate genes among the differentially expressed genes. (b) The three mRNA levels for FN1, CCND2, and ITGB8 on the gene chip. (c) RT-qPCR analyses of FN1 in the A2780 and OVCAR3 cells. GAPDH was used for normalization. Right, melting curves using the real-time PCR target genes (FN1, CCND2, and IGTB8) and the endogenous reference gene.

\section{Discussion}

Epithelial ovarian cancer (EOC) is one of the most common cancers among females and is the fifth leading cause of cancer death among women in the United States with 21,290 new cases and 14,180 deaths in 2015 [14]. Unfortunately, due to the lack of early detection markers and technical skills, most patients are diagnosed in the advanced stage of the disease, which is associated with a very poor survival rate and high levels of metastasis [15-17]. In our paper, we compared two different ovarian cancer cell lines with different migration and invasion abilities and found a new protein marker, FN1, which seems to be a strong candidate marker for the diagnosis of aggressive ovarian cancer. FN1 could also potentially be applied as an indicator of ovarian cancer progression or metastasis.

OVCAR3 and A2780 are the cell lines that are most commonly used in ovarian cancer studies [18-21]. In our laboratory, we found that these two different cell lines exhibit strongly different cell migration and invasion abilities. Transwell and scratch experiments were conducted to confirm these differences. The results revealed that the OVCAR 3 cell line exhibited significantly stronger invasion and migration abilities than the A2780 cell line. To understand the different abilities of these two cell lines, gene chips were used to analyze the differences in gene expression. All are differentially expressed if they met the condition of a fold change greater than 2 or less than -2 with a $P$ value $<0.05$. To narrow the set of differentially expressed genes, stricter conditions were applied for the gene screening. After applying these stricter conditions (cell adhesion related, $P<0.001$, and a fold change of greater than 100 or less than -100), only three genes (i.e., CCND2, ITGB8, and FN1) were found. After the expressions of the CCND2, ITGB8, and FN1 genes in the gene chip were statistically analyzed, the gene that was most strongly correlated with the invasion and migration abilities was found to be FN1. Next, real-time PCR experiments were used to confirm that the OVCAR 3 cells did exhibit higher levels of FN1 expression than the A2780 cells, which exhibited weaker invasion ability than the OVCAR3 cells. And the FN1 KO cell line was used for further confirmation.

FN1 is a core component of many extracellular matrices where it regulates a variety of cell activities through direct interactions with cell surface integrin receptors. FN1 is synthesized by many adherent cells and then assembled into a fibrillation network [2]. Moreover, FN1 expression has been demonstrated to be closely associated with various migration processes, including wound healing, embryogenesis, and 


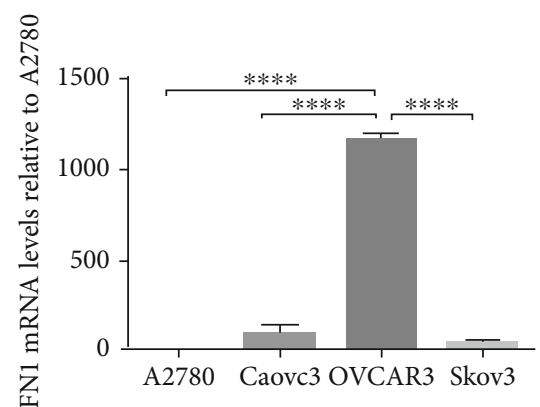

(a)

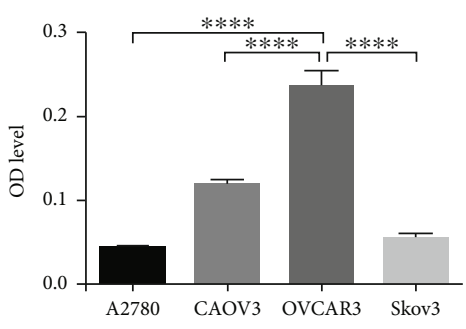

(c)

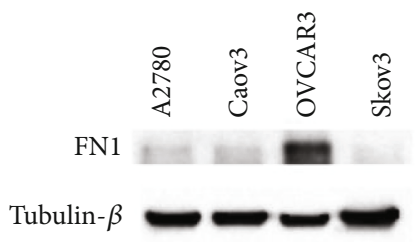

(b)

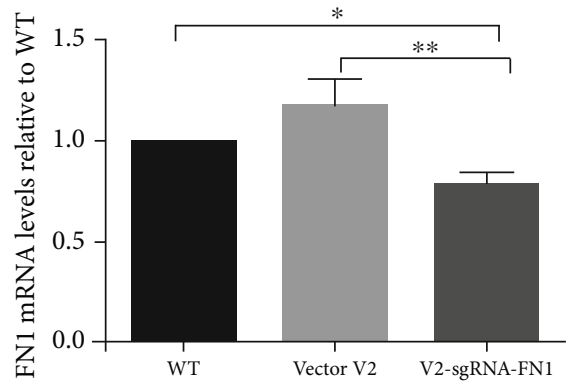

(d)

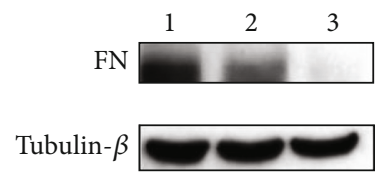

(e)

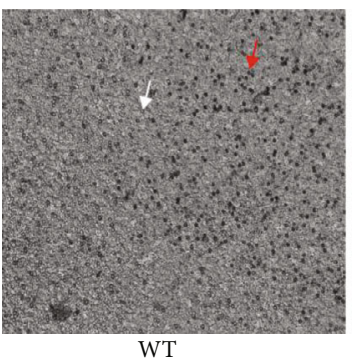

WT

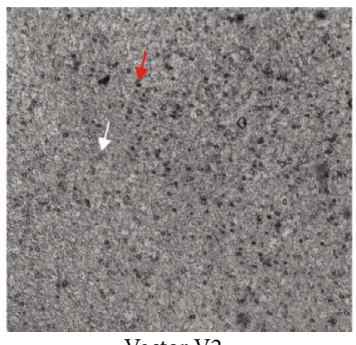

Vector V2

(f)

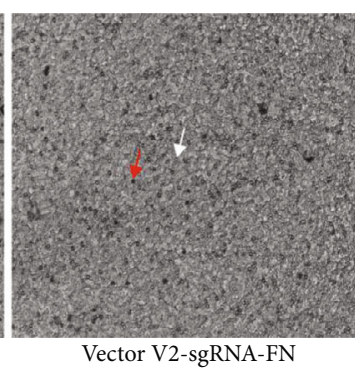

Vector V2-sgRNA-FN

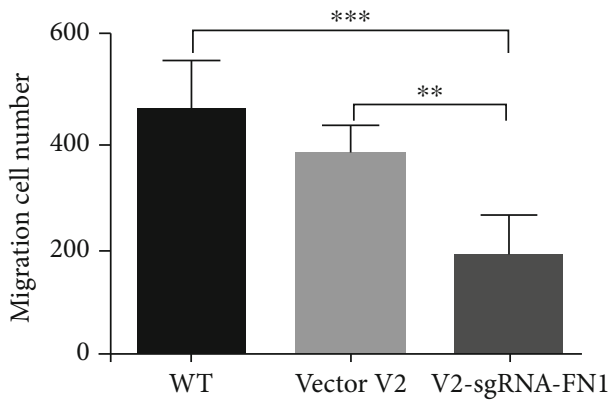

(g)

Figure 4: The FN1 KO cell was used for further confirmation. (a) The mRNA expression of FN1 was tested by real-time PCR among A2780, Caov3, OVCAR3, and Skov3. (b) The cell protein level of FN1 was detected by western blot among A2780, Caov3, OVCAR3, and Skov3. (c) The FN1 level in cell medium was detected by ELISA among A2780, Caov3, OVCAR3, and Skov3. (d) The knockout FN1 mRNA efficiency was detected by real-time PCR among wild-type cell (WT), control cell (vector V2), and the KO cell (V2-sgRNA-FN1). (e) The knockout FN1 protein level efficiency was detected by real-time PCR among wild-type cell (WT), control cell (vector V2), and the KO cell (V2-sgRNA-FN1). (f) Transwell assays were conducted among wild-type cell (WT), control cell (vector V2), and the KO cell (V2-sgRNA-FN1). (g) Quantitative results of the transwell invasion assays. Significant differences were identified using unpaired $t$-test analyses and are indicated by asterisks; ${ }^{* * *} P<0.001$ and ${ }^{* *} P<0.01$. 


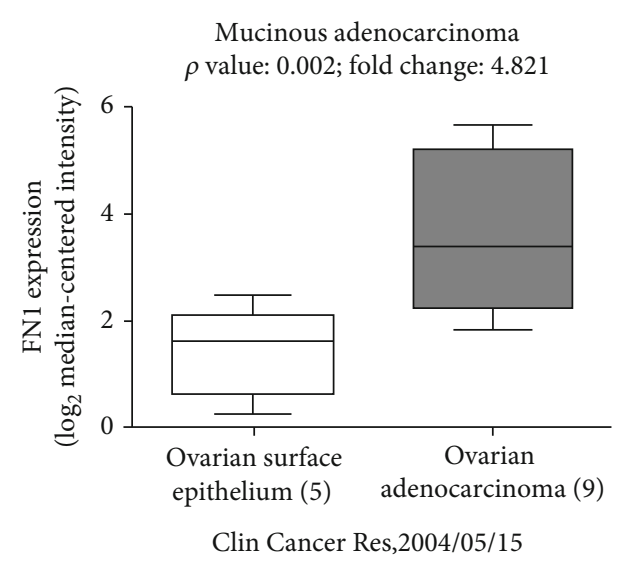

(a)

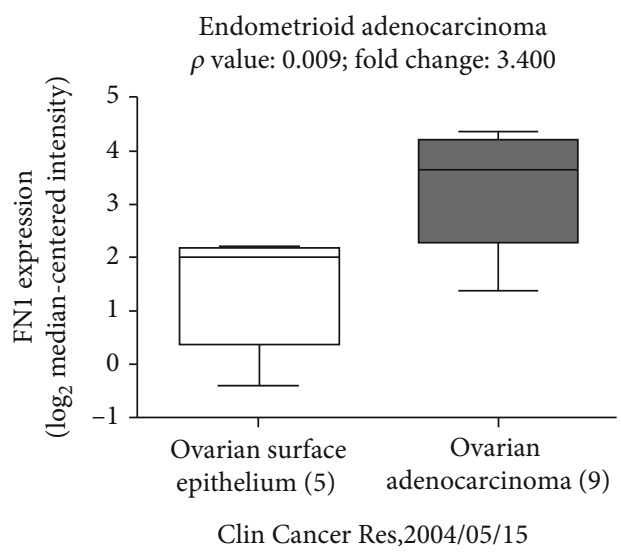

(c)

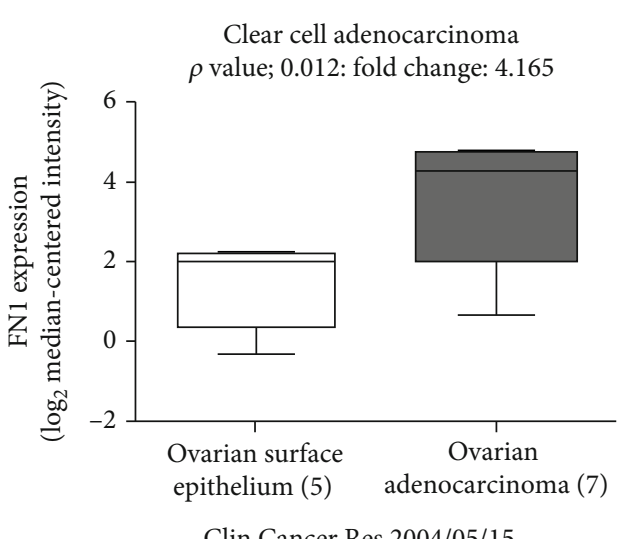

Clin Cancer Res,2004/05/15

(b)

Serous adenocarcinoma $\rho$ value: 0.010 ; fold change: 3.165

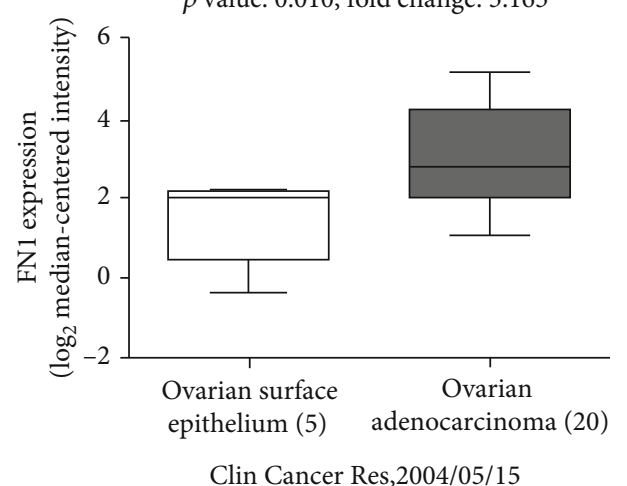

(d)

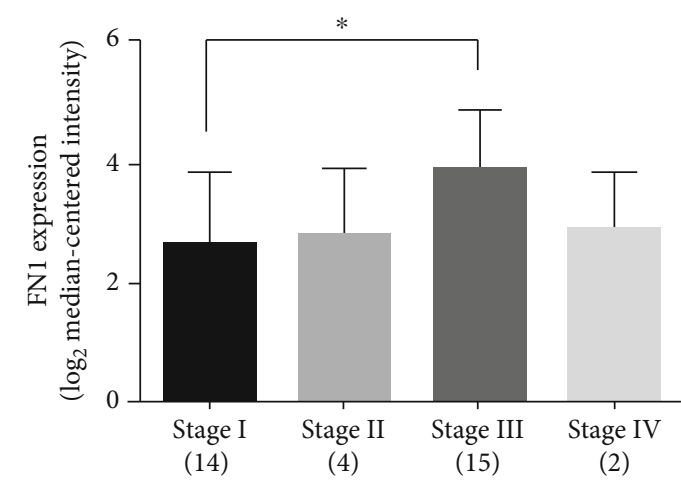

(e)

FIGURE 5: (a-d) Oncomine analyses showing that FN1 is highly expressed in human ovarian mucinous adenocarcinoma, ovarian clear cell adenocarcinoma, ovarian endometrioid adenocarcinoma, and ovarian serous adenocarcinoma. (e) Oncomine analyses showing the expression of FN1 in ovarian cancer according to the different FIGO stages. ${ }^{*} P<0.05$.

metastasis of cancer cells [22]. It has been reported that the extra domain A of FN1 could be a vascular marker of liver and lung metastases. Chen et al. found that both FN1 and TGM2 can facilitate the migration process of A431 tumor cells [23]. Another report also indicated that FN1 can significantly modulate the progression of glioma cells by preserving integrin $\beta 1 \mathrm{FN}$ receptors in glioma cells [24]. Moreover, Franke et al. and Kujawa et al. also observed that fibronectin was an important prognostic factor in ovarian cancer and may be central to tumor progression $[25,26]$. Based on these results, we believe that FN1 could be involved in the progression of ovarian cancer and could be the main reason for the differences in the migration and invasion abilities of these two cell lines. Actually, it has been reported that FN1 can prevent the apoptosis of ovarian cancer cells caused by therapeutic agents. It was suggested that FN1 could be used as a marker to indicate tumor progression in ovarian cancer. This hypothesis was confirmed by the clinical samples. 
In our study, online database analysis (Oncomine) [10] was used to examine the expression levels in patients, and ovarian cancer patients were found to have higher FN1 expression levels than normal subjects. Additionally, there was a significant increase in FN1 expression in stage III cancer compared with lower FIGO stage cancers (stages I and II). Greater FN1 expression was associated with a higher FIGO stage. Both of the results indicated FN1 should be a good marker for ovarian cancer patients.

In conclusion, based on in vitro experiment results and the results from the online database analysis, we believe that FN1 could be used as a marker of ovarian cancer detection and could also be used as a progress indicator for ovarian cancer patients.

\section{Data Availability}

All the data has been presented in the paper.

\section{Conflicts of Interest}

The authors do not have any conflicts of interest.

\section{Acknowledgments}

This work is supported by the scientific research projects of Tianjin Education Commission (2018KJ077).

\section{References}

[1] Y. Xu, L. Chen, B. Xu et al., "Higher numbers of T-bet+ tumorinfiltrating lymphocytes associate with better survival in human epithelial ovarian cancer," Cellular Physiology and Biochemistry, vol. 41, no. 2, pp. 475-483, 2017.

[2] P. C. Buchanan, K. L. M. Boylan, B. Walcheck et al., "Ectodomain shedding of the cell adhesion molecule nectin- 4 in ovarian cancer is mediated by Adam10 and Adam17," The Journal of Biological Chemistry, vol. 292, no. 15, pp. 6339-6351, 2017.

[3] Y.Sun, W. Tao, M. Huang, X. Wu, and J. Gu, "Genetic variants in telomere-maintenance genes are associated with ovarian cancer risk and outcome," Journal of Cellular and Molecular Medicine, vol. 21, no. 3, pp. 510-518, 2017.

[4] H. H. Lee, V. Bellat, and B. Law, "Chemotherapy induces adaptive drug resistance and metastatic potentials via phenotypic Cxcr4-expressing cell state transition in ovarian cancer," PLoS One, vol. 12, no. 2, article e0171044, 2017.

[5] R. Pankov and K. M. Yamada, "Fibronectin at a glance," Journal of Cell Science, vol. 115, no. 20, pp. 3861-3863, 2002.

[6] Y. Mao and J. E. Schwarzbauer, "Fibronectin fibrillogenesis, a cell-mediated matrix assembly process," Matrix Biology, vol. 24, no. 6, pp. 389-399, 2005.

[7] W. Gao, Y. Liu, R. Qin, D. Liu, and Q. Feng, "Silence of fibronectin 1 increases cisplatin sensitivity of non-small cell lung cancer cell line," Biochemical and Biophysical Research Communications, vol. 476, no. 1, pp. 35-41, 2016.

[8] X. Jiang, L. Feng, B. Dai, L. Li, and W. Lu, "Identification of key genes involved in nasopharyngeal carcinoma," Brazilian Journal of Otorhinolaryngology, vol. 83, no. 6, pp. 670-676, 2016.

[9] M. Song, Y. Wang, Z. Zhang, and S. Wang, "PSMC2 is upregulated in osteosarcoma and regulates osteosarcoma cell proliferation, apoptosis and migration," Oncotarget, vol. 8, no. 1, pp. 933-953, 2017.

[10] G. Song, K. Liu, X. Yang et al., "SATB1 plays an oncogenic role in esophageal cancer by up-regulation of FN1 and PDGFRB," Oncotarget, vol. 8, no. 11, pp. 17771-17784, 2017.

[11] X. Lou, X. Han, C. Jin et al., "Sox2 targets fibronectin 1 to promote cell migration and invasion in ovarian cancer: new molecular leads for therapeutic intervention," OMICS, vol. 17, no. 10, pp. 510-518, 2013.

[12] H. Li, N. Xiao, Z. Li, and Q. Wang, "Expression of inorganic pyrophosphatase (PPA1) correlates with poor prognosis of epithelial ovarian cancer," The Tohoku Journal of Experimental Medicine, vol. 241, no. 2, pp. 165-173, 2017.

[13] M. T. Lau, C. Klausen, and P. C. Leung, "E-cadherin inhibits tumor cell growth by suppressing PI3K/Akt signaling via $\beta$ catenin-Egr1-mediated PTEN expression," Oncogene, vol. 30, no. 24, pp. 2753-2766, 2011.

[14] Z. Li, N. Hong, M. Robertson, C. Wang, and G. Jiang, "Preoperative red cell distribution width and neutrophil-tolymphocyte ratio predict survival in patients with epithelial ovarian cancer," Scientific Reports, vol. 7, no. 1, article 43001, 2017.

[15] U. Ray, S. Roy Chowdhury, M. Vasudevan, K. Bankar, S. Roychoudhury, and S. S. Roy, "Gene regulatory networking reveals the molecular cue to lysophosphatidic acid-induced metabolic adaptations in ovarian cancer cells," Molecular Oncology, vol. 11, no. 5, pp. 491-516, 2017.

[16] Y. Jin, X. Xu, X. Wang et al., "Increasing sensitivity to DNA damage is a potential driver for human ovarian cancer," Oncotarget, vol. 7, no. 31, pp. 49710-49721, 2016.

[17] R. H. Berndsen, A. Weiss, U. K. Abdul et al., "Combination of ruthenium(II)-arene complex $\left[\mathrm{Ru}\left(\eta^{6}-\mathrm{p}_{-}\right.\right.$-cymene $\left.) \mathrm{Cl}_{2}(\mathrm{pta})\right]$ (RAPTA-C) and the epidermal growth factor receptor inhibitor erlotinib results in efficient angiostatic and antitumor activity," Scientific Reports, vol. 7, no. 1, p. 43005, 2017.

[18] G. Paragh, S. M. Kumar, Z. Rakosy, S. C. Choi, X. Xu, and G. Acs, "RNA interference-mediated inhibition of erythropoietin receptor expression suppresses tumor growth and invasiveness in A2780 human ovarian carcinoma cells," The American Journal of Pathology, vol. 174, no. 4, pp. 15041514, 2009.

[19] Y.-J. Lee, C.-C. Wu, J.-W. Li et al., "A rational approach for cancer stem-like cell isolation and characterization using CD44 and prominin-1(CD133) as selection markers," Oncotarget, vol. 7, no. 48, pp. 78499-78515, 2016.

[20] B. Li, S. Cai, Y. Zhao et al., "Nerve growth factor modulates the tumor cells migration in ovarian cancer through the $\mathrm{WNT} / \beta$ catenin pathway," Oncotarget, vol. 7, no. 49, pp. 81026-81048, 2016.

[21] M. Zhang, C. E. Gao, W. H. Li et al., "Microarray based analysis of gene regulation by mesenchymal stem cells in breast cancer," Oncology Letters, vol. 13, no. 4, pp. 2770-2776, 2017.

[22] J. Soikkeli, P. Podlasz, M. Yin et al., "Metastatic outgrowth encompasses col-I, Fn1, and Postn up-regulation and assembly to fibrillar networks regulating cell adhesion, migration, and growth," The American Journal of Pathology, vol. 177, no. 1, pp. 387-403, 2010.

[23] S. Sengupta, S. Nandi, E. S. Hindi, D. A. Wainwright, H. Yu, and M. S. Lesniak, "Short hairpin RNA-mediated fibronectin knockdown delays tumor growth in a mouse glioma model," Neoplasia, vol. 12, no. 10, pp. 837-847, 2010. 
[24] H. Zhang, Z. Sun, Y. Li, D. Fan, and H. Jiang, "MicroRNA$200 \mathrm{c}$ binding to FN1 suppresses the proliferation, migration and invasion of gastric cancer cells, migration and invasion of gastric cancer cells," Biomedicine \& Pharmacotherapy, vol. 88, pp. 285-292, 2017.

[25] F. E. Franke, R. Von Georgi, M. Zygmunt, and K. Münstedt, "Association between fibronectin expression and prognosis in ovarian carcinoma," Anticancer Research, vol. 23, no. 5b, pp. 4261-4267, 2003.

[26] K. A. Kujawa, E. Zembala-Nożyńska, A. J. Cortez, T. Kujawa, J. Kupryjańczyk, and K. M. Lisowska, "Fibronectin and periostin as prognostic markers in ovarian cancer," Cell, vol. 9, no. 1, p. 149, 2020. 